УДК 004.5

\title{
ОБЗОРЫ
}

\author{
ГАДЖЕТЫ В ДИСТАНЦИОННОМ ОБРАЗОВАНИИ \\ Иванько А.Ф., Иванько М.А., Ибрагимов А.А. \\ Московский политехнический университет, Москва, е-mail: alekfed@mail.ru, \\ mihaleks@mail.ru,tima.kurkov7@mail.ru
}

\begin{abstract}
Использование смартфонов как устройств связи в обществе стало повсеместным, и оно, как никогда раньше, волнует многих ученых. Но пользование любыми смартфонами должно приносить пользу как в плане инноваций, так и в плане определенных благ, ведущих к процветанию общества в целом. Ученые в последнее время сулят развитие технологий смартфона, это и заметно по последним, постоянно обновляющимся моделям устройств. Необходимо постоянно сохранять современность смартфонов так, чтобы не только отвечать ожиданиям пользователей, но и направлять эту «современность» на развитие цивилизации. Основным направлением в этой статье было изучение данного устройства, которым пользуется достаточное количество людей, с целью разобраться в его влиянии на систему образования и разобраться в возможности использования девайса в дистанционном образовании, что именно помогает смартфону положительно влиять на образование людей. Для того чтобы изучить данный спектр современной науки, была исследована определенная литература, которая поможет сделать определенные выводы о том, что стремительное в последние десятилетия развитие гаджетов является фактором приобретения людьми некоторых знаний о технологиях. Более того, последние модели смартфонов, как показывают некоторые исследования, предстают перед нами главными элементами в уменьшении и в дальнейшем искоренении проблемы провала в знаниях, который в основном возникает в обществе из-за практического отсутствия знаний в области техники, а также выгоды, которую смартфон может приносить обществу.
\end{abstract}

Ключевые слова: смартфоны, развитие образования, инновационные технологии в дистанционном образовании, влияние телефонов, роль смартфонов

\section{GADGETS IN DISTANCE EDUCATION}

Ivanko A.F., Ivanko M.A., Ibragimov A.A.

Moscow Polytechnic University, Moscow, email: alekfed@mail.ru, mihaleks@mail.ru,tima.kurkov7@mail.ru

\begin{abstract}
The use of smartphones as communication devices in society has become ubiquitous, and it, as never before, excites many scientists. But the use of any smartphone should bring benefits, both in terms of innovation and in terms of certain benefits that lead to the prosperity of society as a whole. Scientists have recently promised the development of smartphone technologies, and this is noticeable in the latest, constantly updated models of devices. It is necessary to constantly preserve the modernity of smartphones, so as not only to meet the expectations of users, but also to direct this «modernity» to the development of civilization. The main focus of this article was to study this device, which is used by a sufficient number of people, in order to understand its impact on modern society, whether the smartphone affects us so well, and, perhaps, to see what exactly helps the smartphone to positively influence people. In order to study this spectrum of modern science, a certain literature has been studied, which will help to draw certain conclusions that the rapid development of gadgets in recent decades is a factor in the acquisition of some knowledge by people. Moreover, the latest models of smartphones, as shown by some studies, appear to us as the main elements in reducing and further eliminating the problem of the gap in knowledge, which mainly occurs in society due to the practical lack of knowledge in the field of technology, as well as the benefits that a smartphone can bring to society.
\end{abstract}

Keywords: smartphones, the development of education, innovative technologies in distance education, the impact of phones, the role of smartphones

Информационные технологии в последнее время проходят через все виды человеческой деятельности: учеба, бизнес, различные путешествия, общение в социальных сетях. Смартфоны - это самое удобное устройство, которое используется людьми для получения информации. От проводных телефонов до более продвинутых и навороченных устройств, таких как смартфоны, на протяжении этого времени технологии, не снижая темпа, все совершенствовались и совершенствовались, упростив людям общение между собой (уменьшилось время передачи, и, в принципе, теперь, чтобы пообщаться, нужно просто достать телефон из кармана) [1]. Сейчас в маленьком телефоне может быть очень много функций, он может сочетать в себе и фотоаппарат, и видеокамеру, и лист бумаги, на котором можно рисовать. Спектр функций огромен. Смартфоны - это устройства, подобные телефонам, но в отличие от них имеют расширенные опции, выполняют более обширный диапазон запросов пользователя, нежели классические телефонные аппараты, к примеру осущест- 
вление простых телефонных переговоров либо отправление СМС. Они имеют более широкие возможности: показ видеорядов, пользование электронной почтой, производство фото [2]. Смартфоны изначально были предназначены для использования бизнесменами в своем деле из-за их стоимости и незнания, как ими пользоваться, но сегодня существует множество брендов смартфонов разного ценового спектра и различных вариантов моделей, в соответствии с предпочтениями людей. Сейчас смартфоны служат неким связывающим элементом между целевыми пользователями изделий. Также они могут использоваться для общения в разных областях деятельности. Смартфон похож на некий компьютер, только уменьшенную его копию, потому что в данной версии он работает практически как ПК и является портативным устройством для любых желаний пользователей [3]. Развитие технологии разработки смартфона особенно важно в наше время для правильного использования этих устройств нами - людьми. Мир, в котором и так всегда происходят перемены, можно улучшить путем использования полученных навыков и опыта в развитии. Прошлые исследования по данной тематике описывали технологию создания смартфонов без какого-либо обоснования главного условия, который удерживал на отличном уровне формирование новых телефонов и то, какая выгода будет, если рассматривать его как элемент для совершенствования людей.

\section{Цели исследования}

1. Одна из целей статьи состоит в том, чтобы исследовать эту новую стратегию, которой владеют создатели смартфонов, и то, как хорошо это может влиять на общество, которое направлено на свое развитие.

2. Необходимо выяснить, используется ли смартфон в дистанционном образовании, и определить его влияние на данный формат обучения.

3. Показать, насколько за последние несколько лет были усовершенствованы смартфоны.

\section{Материалы и методы исследования}

Для начала в этой статье будет рассмотрена история зарождения и развития смартфонных технологий и их поэтапное развитие до нынешних грандиозных масштабов, также нельзя не сказать про мастодонтов смартфонных технологий, таких как Samsung, Apple, которые, безусловно, посредством продаж своих продуктов влияют на развитие человеческого поколения.
Теория разрыва в знаниях тоже является предметом, который необходимо рассмотреть в этой статье. Также, обозревая некоторые исследования в данной области, рассмотрим, как именно новейшие технологии влияют на обычное образование и дистанционное образование.

\section{Экскурс в историю смартфона}

C IBM Simon Personal Communicator и Nokia 9000 Communicator, в которые уже на тот момент, в девяностые, были встроены функции настоящих смартфонов, началась история современных смартфонов, идея которых, правда, зародилась еще в 70-х годах прошлого века. Но их все еще нельзя было называть смартфонами, хоть у них и имелись такие функции, как отправка электронной почты, факсов и т.п., более того, в некоторых моделях, выпускаемых в то время, уже была возможность выходить с них во Всемирную паутину. Далее с 2000 г. смартфоны развивались и развивались, включая все новые и новые функции в себя, но в 2007 г. произошла настоящая революция в данной сфере, так как компания Apple под руководством Стива Джобса выпустила по-настоящему революционную модель, ту модель, без которой мы сейчас вообще не представляем смартфон как таковой, у новенького iPhone был полностью сенсорный экран [4]. Также данный смартфон на тот момент имел и другие функции, кроме сенсорного экрана, чем сейчас уже точно не удивить пользователей, в нем была возможность скачивать многочисленные приложения из Интернета, он обладал открытой ОС, интеграция датчиков - так называли данный смартфон некоторые научные исследования в данной области $[5 ; 6]$. Все знают, что сейчас смартфон есть, можно сказать, практически у каждого человека (может быть, даже не один такой гаджет), каждый может выйти в Интернет, провести, к примеру, прямую трансляцию, сделать видеозвонок, но в начале своего пути смартфон предназначался, в большинстве своем, для осуществления и поддержания бизнеса, соответственно им пользовалось очень ограниченное число потенциальных пользователей. В общем, смартфон, все более развиваясь, стал очень продвинутой маленькой машинкой, которая помогает нам и облегчает наши жизни, но что можно сказать насчет полезности этого устройства, способно ли оно не только облегчать жизнь, но и нести пользу посредством этих облегчений, может ли человек добывать необходимую ему информацию в телефоне? Человек постоянно должен «питаться» 
информацией, посредством использования смартфона это определенно выполнимо, но это должно происходить на перманентной основе. Из представлений и исследований некоторых ученых ясно, что данные технологии точно будут развиваться и дальше, у смартфонов каждые полтора года будут усложняться методы и схемы их сборки, включаться все новые и новые функции. Многие люди сейчас не представляют свои жизни без этого маленького устройства в своих руках, но если у человека нет определенной цели в использовании смартфона, да, он покупает его, но начинает неправильно использовать его уже с первых минут, что может привести к определенным негативным последствиям. Знания можно назвать силой человечества над другими формами жизни, соответственно, они являются силой любого существующего общества, знания постоянно дополняются, и если отдельно стоящее от других общество отстает от таких высоких стандартов, оно может потерять свою скорость развития и в конце концов развалиться и уйти в небытие [7]. Необходимо, чтобы скорость приобретения информации и улучшение смартфонов были синхронными. Гаджет должен стать для каждого человека основой, позволяющей быстрее соединиться с миром в поисках жизненно важной информации, которая может быть полезна для пополнения запаса знаний, а не информации, которая должна снижать уровень приобретаемых и приобретенных знаний, необходимых для общества. Некоторые ученые пытались взглянуть на разработку новых телефонов, и таким образом они предлагают некоторое ценное высказывание: слово «технология» включает в себя теоретические и практические навыки и вещи, которые могут быть использованы для разработки систем взаимодействия между машиной и человеком. Кроме того, смартфоны всегда были связаны с прогрессом общества, с улучшением его уровня жизни [8]. Получение знаний - это «длительный процесс обратной связи, его стабильность зависит от достоверности полученной информации». Кроме того, люди используют полезные знания, полученные для улучшения своей жизни [9]. Опять же, большая часть используемой сегодня информации находится в Интернете, и, конечно же, предполагается, что изначально будет исследован уровень знаний, приобретаемых обществом. И действительно, вышеупомянутые исследователи подошли к описанию технологии с разных точек зрения, но такой же целью является и рост знаний, связанный с каждым субъ- ективным мнением. Это ли не является показателем, что любое общество должно понимать специфику любой используемой технологии? По сути, технологии разработки смартфонов управляют миром в настоящее время и будут продолжать так же сильно влиять на общество в далеком будущем. Очень важно, чтобы люди уделяли время новой ранее не существовавшей ветви получения знаний, а не последователям данных технологий, и каждое общество, независимо от его размеров, должно больше сосредоточиться на том, чтобы быть объективными пользователями, уменьшающими уровень социальной бедности и превращающими бедное общество в богатое и здоровое. Но есть большой вопрос, который все же следует задать. Он заключается в том, что интеллект в смартфонах параллельно растет с приобретением знаний или же это приведет к еще большему разрыву знаний в обществе. Чтобы ответить на этот вопрос, необходимо рассмотреть технологическую цель некоторых смартфонов, разработанных крупными игроками на рынке смартфонов. Видно, что у создания любых разработанных для технических коммуникаций гаджетов существует не только коммерческая цель, но и общая благая цель. Способность любого человека принять основную технологическую цель технической компании для начала такого развития облегчит нахождение нужной информации в его повседневной деятельности. Все большее и стремительное улучшение технологий создания смартфонов, а также увеличение количества и качества встроенных функций со стороны компаний-гигантов можно принять как гонку за большей прибылью и выгодным местом на рынке производителей. Соответственно, улучшая технологии, компании повышают цены на свои продукты, к слову, сейчас последние модели iPhone стоят более 100 тыс. рублей, это не нравится многим членам общества, что в итоге приводит к необъективности пользования устройствами данных компаний, люди просто начинают гонку за новенькими моделями, чтобы показать свое превосходство, естественно, не настоящее, над другими. Последние модели смартфонов имеют очень большой процент интеграции со стационарными компьютерами, так, например, выпуск iPhone в 2015 г. показал очень большой прогресс в данной области. Более того, была разработана специальная среда для того, чтобы улучшить и облегчить данную интеграцию, чтобы пользователи могли спокойно пользоваться компьютером через свои телефоны. Например, 
акселерометр, позволяющий определять местоположение и движения человека, появился уже в Nokia 5500, а гироскоп, позволяющий определить движения своего владельца, появился в далеком уже 2007 г. в том самом iPhone первого поколения. Подобные функции появляются и обновляются все чаще, они являются интеллектуальными возможностями данных смартфонов, поэтому способствуют и все ускоряющемуся развитию общества. Инновационной стратегией сейчас обладают не многие компании, в большинстве ей владеют компании-мастодонты, такие как Apple, Xiaomi, Huawei и другие, данная стратегия и влияет на то, что компании создают все более сложные и навороченные устройства. Следовательно, именно поэтому данные компании, заполучив эту стратегию уже очень давно, являются теми, кто создает больше всего этих умных устройств. Чтобы получить большую выгоду от использования смартфонов, то есть чтобы получение полезных знаний от его использования все больше и больше увеличивалось, необходимо, чтобы общество не теряло ту инновационную стратегию, которая это все обеспечивает. Её можно описать как «приверженность к ряду последовательных, взаимно использующихся стратегий и действий, направленных на достижение конкретной цели» [10]. Инновационная стратегия очень полезна, ведь она может, несомненно, повлиять на развитие нашего общества, но если она применяется в соответствии с используемыми технологиями, этого не было описано в определении выше, что делает его не очень полным. Доступность к новейшим умным гаджетам, например iPhone, iPad, Mac, является довольно высокой, но если в стране уровень жизни находится на нормальном уровне, сейчас даже в некоторых странах третьего мира гражданин может позволить себе купить данные гаджеты. Некоторые исследования показывают, что сейчас в развивающихся странах смартфоны в основном используются для общения в чатах, так как люди больше всего времени проводят как раз-таки в социальных сетях. Поэтому многие могут бездумно общаться с друзьями часами, а то и днями, не пополняя багаж своих знаний, что может влиять на развитие самого человека, а значит также влиять и на общество как систему. Но все же есть и положительные моменты, например студенты посредством общения в таких дружественных чатах могут обмениваться какой-либо полезной информацией, повышающей их уровень знаний. Поэтому можно сделать вывод, что использование умных устройств не всегда приводит к разрыву в знаниях, это будет рассмотрено дальше.

\section{Описание теории разрыва в знаниях}

В 1970 г. учеными была представлена теория разрыва в знаниях. Предложенная теория отражала, как могут создаваться пробелы в знаниях людей и всего общества в зависимости от статуса человека и его семьи. Из этого следует, что люди богатые способны быстрее потреблять и усваивать все быстрее нарастающую информацию в Интернете и других средствах массовой информации, нежели чем бедные, из-за того что бедные редко могут себе позволить обеспечить вокруг себя некое информационное поле, страдает все общество в целом, из-за этого и возникает разрыв в знаниях во всем обществе. Исследователь, изучающий население Соединенных Штатов Америки в 2009 г., в своей работе пришел к выводу, что между людьми с высоким статусом и людьми с низким есть некоторое сходство. Сходство наблюдается в том, что если информация неинтересна людям, то они перестают отправлять поисковые запросы, то есть перестают искать информацию в принципе. Причинами может служить следующее:

1) у людей пропадает необходимость в поиске информации;

2) если люди сталкиваются с уже существующими знаниями, то им порой бывает сложно усваивать все больше и больше новой информации [11].

Казалось бы, развитие технологий и появление новых средств массовой информации должны были полностью устранить существующий разрыв в знаниях, но, к сожалению, все же расширили его. Сейчас пользователям ежедневно приходит тысячи и тысячи сообщений с абсолютно ненужной им информацией, что, конечно же, отвлекает их от поисков необходимой полезной информации, тем самым они могут еще и запомнить ненужные сообщения-пустышки [12]. Поиск необходимой информации начинается с какой-то личной мотивации пользователя, также на это могут повлиять индивидуальные качества человека и, может быть, даже уровень образованности. Используя доступные технологии смартфона, люди не всегда знают, как правильно пользоваться данным устройством, получая меньшее количество информации, человек не сможет выполнять правильные действия по отношению к обществу, что в свою очередь может привести к непоправимым процессам в обществе, которые могут разрушить его. 
Использование смартфона в роли инструмента обучения

Как мы знаем, образование является двигателем любого развивающегося общества, образование развивает людей, делает их намного умнее и смышленее, что приводит к совершенствованию общества, следовательно, повышению качества и уровня жизни населения планеты, устраняя многие проблемы общества. Образование так же необходимо, как и экономика, без него этой экономики, как и других сфер жизни, существовать попросту не будет, а если и будет, то в ужаснейшем состоянии. С появлением смартфонов, как было упомянуто выше, меняются все сферы деятельности и жизни человека, это не могло обойти и образование. Технологии определенно помогают людям наладить взаимодействия в изучении и обмене знаниями, учителя и ученики могут обмениваться нужной для них информацией, чтобы ускорить процесс образования. Во Всемирной паутине достаточно легко находятся учебные пособия для их дальнейшего изучения, что поддерживает в тонусе обучение людей, поддерживая непрерывность образования. Существование виртуальных библиотек данных, конечно, может привести к отрицанию очных лекций, так как многим начинает казаться, что и там и там преподается один и тот же материал, который можно просто прочитать дома, не ходя в университет $[13 ; 14]$. Однако использование смартфона расслабляет человека и не напрягает его, поэтому некоторые тяжело воспринимаемые предметы могут усваиваться легче, чем при обыденных лекциях, также нередко использующиеся интерактивные вставки делают процесс приобретения знаний гораздо интереснее. Навыков, получаемых от использования технологий, становится все больше и больше с течением времени. Смартфоны повышают навыки человека в использовании технологических приспособлений, что может выработать положительное отношение к технологиям в целом. Как мы знаем, пользователи могут быть разными, именно поэтому технологии способны подстраиваться под каждого из нас, человек может выставить предпочтительные только ему настройки своего гаджета, сделать так, чтобы ему удобнее было воспринимать и усваивать получаемую информацию. Благодаря смартфонам можно сделать очень многие вещи, в них можно устанавливать большое количество необходимого для работы или отдыха софта, делая свою жизнь немного удобнее [15]. Все технологии позволяют пользователям расширять свои знания в области своей профессии или учебы, обмениваясь информацией, люди становятся более образованными и общительными.

\section{Использование смартфонов в дистанционном образовании}

Как уже говорилось выше, смартфоны имеют в себе достаточное количество функций, позволяют своим пользователям свободно работать, учится и развлекаться, где бы они ни находились, поэтому данные устройства уже давно стали частью нашей жизни. Достаточно большое количество обучающихся в университетах студентов имеют совершенно новые модели смартфонов и уже даже являются большими профессионалами в их использовании, например в навигации при помощи GPS, использовании множества сервисов (Google Chrome, What's App), которые помогают в коммуникации и в осуществлении обучения, в том числе дистанционного. Все больше и больше людей подключаются к услугам мобильного интернета, именно поэтому использование сервисов возрастает очень быстро. Педагог Скотт П. Симкинс имеет свое мнение по поводу использования технологий в дистанционном образовании, он считает, что тут важнее не педагогика, а то, как инновации в педагогике используются в соответствии с той средой, где осуществляется обучение [16]. Сделать обучение более доступным для студентов, обеспечив их необходимыми электронными курсами, является главной целью дистанционного образования, а смартфоны, несомненно, могут помочь в этом. Благодаря встроенным функциям смартфоны могут обеспечить бесперебойный доступ к курсам дистанционного обучения, организованным в высшем учебном заведении, поэтому они так популярны среди студентов. Когда ученик может выбрать свой, более удобный для него вид устройства, дистанционное обучение по программам будет гораздо продуктивнее. В основном многие выбирают именно смартфоны, ведь они намного мобильнее, чем те же ноутбуки. С появлением в образовании смартфонов становится гораздо проще получить те навыки, которые необходимы студенту в будущем на его работе [17]. Дистанционное обучение, несомненно, с каждым годом становится все больше распространенным в мире способом получения знаний, и данные технологии порой ничуть не хуже традиционных видов обучения. Не показывая людям, что дистанционное обучение является вполне хорошим способом получения знаний, а также не обеспечивая людей необходимой техникой, можно сказать, что эффективность дистанционного образования отсутствует [18; 19]. Многие исследования 
в данной сфере, где сравнивается дистанционная форма с традиционной, показывают, что результат обучения в дистанционном образовании зависит, прежде всего, от самодисциплины студента. Курсы дистанционного обучения успешны тогда, когда студент имеет мотивацию, подкрепленную тем, что он хочет удовлетворить свои потребности в получении знаний, а также достичь своих целей в этой сфере [20]. Поэтому руководство и преподаватели университета должны найти подход к ученикам, найти то устройство, которое больше всего обеспечивает мотивацию студента обучаться по образовательным программам. В последнее время в дистанционном образовании существуют не только курсы, но и видеоконференции. Многие исследователи считают, что общение преподавателя со студентами в формате вебинара добавляет в образование общение с преподавателем без присутствия при этом в аудитории, также данный формат может помочь студентам легче найти общий язык со своими коллегами [21]. Сейчас технологии развиты настолько, что даже с помощью смартфонов возможно присутствовать на видеоконференциях. Стационарные компьютеры и даже ноутбуки, хотя, казалось бы, они тоже довольно мобильные, все меньше и меньше используются и студентами, и преподавателями. Безусловно, преподаватели заинтересованы в обеспечении комфортного онлайн-обучения и для себя, и для студентов, тем самым они скачивают необходимый для этого софт, например Zoom, Webinar и др. - все это может обеспечить отличное соединение и занятие для всех. Так, с помощью данных сервисов можно и читать лекции, и проводить практические занятия, и даже сдавать дипломные и другие зачетные работы перед всей группой или потоком. Большинство студентов уже сейчас не хотят приобретать себе ноутбук, так как даже самые новые модели весят достаточно много, по крайней мере больше, чем смартфон. Именно поэтому ученики и приобретают смартфоны, ведь они являются сейчас полной заменой ПК. Сейчас большинство ПО, которое раньше было доступно только в десктоп-версии, перекочевывает в телефоны, например уже сейчас в Play Market или App Store можно бесплатно скачать и пользоваться программами из MS Office и практически с полным функционалом пользоваться ими. Для видеоконференций преподаватели и руководители, специализирующиеся на дистанционном образовании, преимущественно выбирают те приложения, которые возможно установить и на мобильные ОС. Смартфоны сейчас очень широко начинают использовать в дистанци- онном образовании. Многие исследователи изучают данную сферу, введение смартфонов в дистанционное образование, оценивая вклад смартфонов [22-24]. Понятно, что мобильное оборудование в виде маленького устройства - смартфона, обеспечивает обучение в любое время и в любом месте, естественно, нужно только позаботиться о зарядке и выходе в Интернет, но это тоже не проблема в наши дни, ведь сейчас очень обширен сегмент мобильного интернета, а для зарядки смартфона можно использовать Power Bank. В некоторых исследованиях приводятся факты того, что преподаватели все больше сами используют КПК и остальные мобильные устройства в электронном образовании, также преподаватели записывают подкасты для своих студентов, тем самым предоставляя им возможность сохранить для себя данные записи, чтобы, например, подготовиться к экзамену или зачету. В дальнейшем ученики могут поддерживать обратную связь с преподавателем, используя при этом все также смартфон. Многие высшие учебные заведения сейчас имеют свои системы дистанционного образования, но если таковой нет, то есть множество других программ в Интернете. Такими системами можно также спокойно, а главное удобно пользоваться при помощи смартфона [25; 26]. Прежде не было упоминания о возможности сдавать экзамены через смартфоны, но в данный момент, из-за сложившейся сложной ситуации с пандемией, вузы вынуждены проводить и защиту дипломов, и обычные экзамены и зачеты дистанционно.

\section{Результаты исследования и их обсуждение}

В исследовании изучалась важность развития, а самое главное, принятия технологий смартфонов всеми людьми, так как в основном данные технологии приносят больше пользы, чем вреда для людей. Гораздо лучше технологии влияют на образование, ведь именно в образовательных целях и нужно больше всего использовать данные технологии, обмен информацией через Сеть очень ускоряет процесс обучения человека. Была рассмотрена теория провала в знаниях, и ясно, что при уменьшении количества принимаемой информации в конце концов следует развал общества. Понятно, что в дистанционном образовании смартфон играет огромную роль, он существенно упрощает и жизнь студентов, и жизнь преподавательского состава, предоставляя им возможность не сидеть постоянно за ноутбуком или стационарным компьютером, и в любое время и в любом месте решать 
свои проблемы по учебе. Смартфон - это, в принципе, очень хорошее подспорье человеку, которое, несомненно, упрощает и улучшает его жизнь в плане скорости работы, но и то устройство, которое может захватить все время человека, если, конечно же, он будет неправильно использовать гаджет.

\section{Список литературы}

1. Klein E. Technology is changing how we live, but it needs to change how we work. Vox. 2012. [Электронный pecypc]. URL: https://www.vox.com/a/new-economy-future/technology-productivity (дата обращения: 09.05.2020).

2. Sarwar M., Soomro T.R. Impact of Smartphone's onsociety. European Journal of Scientific Research. 2013. no. 98. P. 216-226.

3. Rambitan V.M. The effect of smartphone on students'critical thinking skill in relation to the concept of biodiversity.Am. J. Edu. Res. 2015. no. 3. P. 243-249.

4. Martin T. The evolution of the smartphone. Pocketnow. 2014. [Электронный ресурc]. URL: https://pocketnow. com/the-evolution-of-the-smartphone (дата обращения: 09.05.2020).

5. Miyashita Y. Evolution of mobile handsets and theimpact of smartphones. InfoCom Research, Inc. 2012. P. 2-31. URL: https://www.icr.co.jp/docs/Evolution of Mobile Handsets_and the_Impact_of_Smartphones.pdf (дата обращения: 09.05.2020).

6. Campbell A., Choudhury T. From smart tocognitive phones. IEEE Pervasiv Computing. 2012. no. 11. P. 7-11.

7. Braunerhjelm P. Entrepreneurship, innovation and economic growth past experiences, current knowledge and policy implications. Swedish Entrepreneurship Forum. 2010. P. 3-45.

8. Dolinsek S., Strukelj P. Technology, wealth and modern management of technology. Managing Global Transitions International Research Journal. 2012. no. 10. P. 29-49.

9. Pentzaropoulos G.C. New technologies, the Infoworld and the need for actionable knowledge. Electronic Journal for Philosophy. 2016. no. 22. P. 51-61.

10. Pisano G.P. You need an innovation strategy. Harvard Business Review. 2015. no. 93. P. 44-54.

11. Chen X. The influence of social media on knowledge gaps about science and technology among Chinese audiences. Graduate Thesis, Iowa State University, Iowa. 2013. P. 1-32.
12. Anaeto S.G., Onabajo O., Osifeso J.B. Models and Theories of Communication. African Renaissance Books Incorporated, Senegal. 2008.

13. Sanusi B.O., Adelabu O., Okunade J.K. Adapting social media for formal learning in Nigeria: Challenges and prospects. Arabian J. Bus. Manage. Rev. 2014. no. 3. P. 22-30.

14. Cifuentes O.E., Lents N.H. Increasing student-teacher interactions at an urban commuter campus through instant messaging and online office hours. Electron. J. Sci. Educ. 2011. no. 14. P. $1-13$.

15. Bansal T., Joshi D. A study of students' experiences of mobile learning. Global J. Hum. Soc. Sci. 2014. no. 14. P. 26-30.

16. George S.B. Towards Innovative Pedagogy. The Hindu: Education Plus. 2014, January 20. P. 1-4.

17. Rajesh M. Revolution in Communication Technologies: Impact on Distance Education. Turkish Online Journal of Distance Education. 2015. vol. 1. no. 16. P. 62-88.

18. Tuncay N., Poyraz C. Distance Education from «Impossible» To Be «Possible». Journal of Educational and Instructional Studies in the World. 2013. vol. 2. no. 3. P. 143-154.

19. Tuncay N., Öznacar M.D. Sanal Düşler ve Özel Gerçekler: Özel Eğitim Gerektiren Bireyler ve Uzaktan Eğitim, Anı Yayıncılık: Ankara. 2014. P. 3-23.

20. Goulimaris D. The Relation between Distance Education Students' Motivation and Satisfaction, Turkish Online Journal of Distance Education 2015. vol. 2. no. 16. P. 13-27.

21. Lim S., Cha S., Park C., Lee I., Kim J. Getting closer and experiencing together: Antecedents and consequences of psychological distance in social media enhanced real-time streaming video. Computers in Human Behaviour. 2012. vol. 4. no. 28. P. $1365-1378$.

22. Nawi M.A.M, Jamsari E.A., Hamzah M.I., Sulaiman A., Umar A. The Impact of Globalization on Current Islamic Education. Australian Journal of Basic and Applied Science. 2012. vol. 8. no. 6. P. 74-78.

23. Shuib A.S. Reka Bentuk Kurikulum M-Pembelajaran Sekolah Menengah: Teknik Delphi; Proceedings of Regional Conference on Knowledge Integration in ICT. 2010. P. 652-665.

24. Nawi A., Hamzah M.I., Rahim A.A. Teachers Acceptance of Mobile Learning for Teaching and Learning in Islamic Education: A Preliminary Study, Turkish Online Journal of Distance Education. 2015. vol. 1. no. 16. P. 184-192.

25. Bell B. Theorising teaching in secondary classrooms: Understanding our practice from a sociocultural perspective. N.Y.: Routledge. 2011. P. 65-98.

26. Dianne Forbes \& Elaine Khoo. Voice over distance: a case of podcasting for learning in online teacher education, Distance Education. 2015. vol. 3. no. 36. P. 335-350. 